CORRESPONDENCE

\title{
SARS-CoV-2-specific T cell immunity to structural proteins in inactivated COVID-19 vaccine recipients
}

\author{
Yao Deng ${ }^{1,3}$, Yu Li ${ }^{2,3}$, Ren Yang (iD ${ }^{1}$ and Wenjie $\operatorname{Tan}^{1 凶}$ \\ (C) The Author(s), under exclusive licence to CSI and USTC 2021 \\ Cellular \& Molecular Immunology (2021) 18:2040-2041; https://doi.org/10.1038/s41423-021-00730-8
}

In patients recovering from coronavirus disease 2019 (COVID-19), specific T-cell responses against severe acute respiratory syndrome coronavirus 2 (SARS-CoV-2) have been detected [1]. These responses targeted not only the spike (S) protein but also the membrane $(\mathrm{M})$, nucleocapsid $(\mathrm{N})$, and other open reading frames (ORFs) [2]. A licensed inactivated COVID-19 vaccine in China, BBIBP-CorV, induced good humoral responses [3]. However, whether virus-specific $T$ cell immunity could be induced by BBIBP-CorV has not yet been clarified. Given that recent studies have demonstrated that mRNA (BNT162b2, mRNA-1273) and viral vector vaccines elicit strong $T$ cell responses as well as neutralizing Abs against SARS-CoV-2 [4], we conducted a study to evaluate the T-cell response to SARS-CoV-2 before and after receiving BBIBPCorV.

The study was approved by the Institutional Review Board of the China CDC. All vaccinees provided written informed consent. We collected blood samples from 10 healthy individuals before and after vaccination (median age, 35.5 years; range, 23-51; 3 males and 7 females) who received BBIBP-CorV containing $4 \mu \mathrm{g}$ of total protein on days 0 and 21. Postvaccination blood was collected 12 weeks after the second dose. We performed IFN-y ELISpot assays on PBMCs to quantify the frequency of virusspecific $T$ cells (details are provided in the Supplementary Appendix, available with the full text of this letter). A significant increase in IFN- $\gamma$ spot-forming units (SFU) per million peripheral blood mononuclear cells (PBMCS) was observed against the $\mathrm{S}, \mathrm{N}$ and $E$ peptide pools of SARS-CoV-2 after vaccination (Fig. 1A-C). Nevertheless, the increase was not statistically significant for the $M$ peptide pools, although the increase in IFN- $\gamma$ SFU per million PBMCs was observed in six out of the 10 study participants (Fig. 1D). Given the presence of a certain degree of homology in the amino acid sequence of epitope peptides between SARS-CoV2 and endemic HCoVs, preexisting SARS-CoV-2-reactive T cells or cross-reactivity with HCoVs might be detected in certain individuals; [5] this was observed in the significant SFU background in the prevaccination samples. We also tested postvaccination serum samples for lgG antibodies against the $S$ protein (details on the laboratory assays are provided in the Supplementary Appendix). All study participants had detectable IgG antibodies with a median titer of 1:80 (range: 1:10 to 1:160). There was no significant correlation between the IgG antibody titer and IFN- $\gamma \mathrm{SFU}$ against the $\mathrm{S}$ protein (Spearman's correlation coefficient $\rho=-0.53, P=0.11$ ).
Our findings demonstrate that specific $T$ cell responses to multiple structural proteins ( $\mathrm{S}, \mathrm{N}$, and $\mathrm{E}$ proteins) of SARS-CoV-2 were elicited in BBIBP-CorV recipients. This suggests that inactivated vaccines against SARS-CoV-2 may elicit T-cell responses in addition to humoral responses, and cellular responses may play a role in the protection offered by inactivated vaccines. Our findings also suggest that there is likely no association between vaccine-induced $T$ cell responses and humoral immunity. The limitations of this study were the limited number of study participants and lack of a phenotypical characterization of the SARS-CoV-2 T-cell response. Further experiments will be very helpful for characterizing the T-cell subsets and their responses to SARS-CoV-2 in a larger sample of participants. It is important to determine the immune correlates of protection against SARS-CoV-2 infection.

\section{REFERENCES}

1. Chen Z, John Wherry E. T cell responses in patients with COVID-19. Nat. Rev. Immunol. 2020;20:529-36. John Wherry. 2020

2. Cassaniti I, Percivalle E, Bergami F, A Piralla, G Comolli, R Bruno, et al. SARS-CoV-2 specific $T$ cell immunity in COVID-19 convalescent patients and unexposed controls measured by ex-vivo ELISpot assay. Clin Microbiol Infect. 2021. S1198-743X (21)00145-2.

3. Xia $S$, Zhang $Y$, Wang $Y$, Wang $H$, Yang $Y$, Gao GF, et al. Safety and immunogenicity of an inactivated SARS-CoV-2 vaccine, BBIBP-CorV: a randomised, double-blind, placebo-controlled, phase 1/2 trial. Lancet Infect. Dis. 2020;21:39-51.

4. Prendecki M, Clarke C, Brown J, Cox A, Gleeson S, Guckian M, et al. Effect of previous SARS-CoV-2 infection on humoral and T-cell responses to single-dose BNT162b2 vaccine. Lancet. 2021;397:1178-81.

5. Braun J, Loyal L, Frentsch M, Wendisch D, Georg P, Kurth F, et al. SARS-CoV-2reactive $T$ cells in healthy donors and patients with COVID-19. Nature. 2020;587:270-4.

\section{ACKNOWLEDGEMENTS}

We thank all vaccine recipients for providing blood and PBMC samples. This work was supported by grants from the National Natural Science Foundation of China (82041041, 82061138008).

\section{AUTHOR CONTRIBUTIONS}

WJT and YD conceived of the study. YD and RY performed the experiment; YD, YL, and WJT analyzed the data; WJT and YL drafted the manuscript. All authors reviewed and approved the final manuscript.

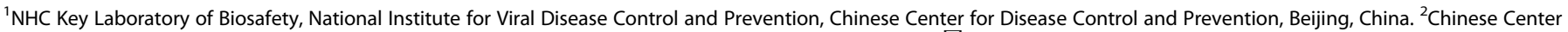
for Disease Control and Prevention, Beijing, China. ${ }^{3}$ These authors contributed equally: Yao Deng, Yu Li. ${ }^{凶}$ email: tanwj@ivdc.chinacdc.cn

Received: 6 June 2021 Revised: 18 June 2021 Accepted: 19 June 2021

Published online: 15 July 2021 

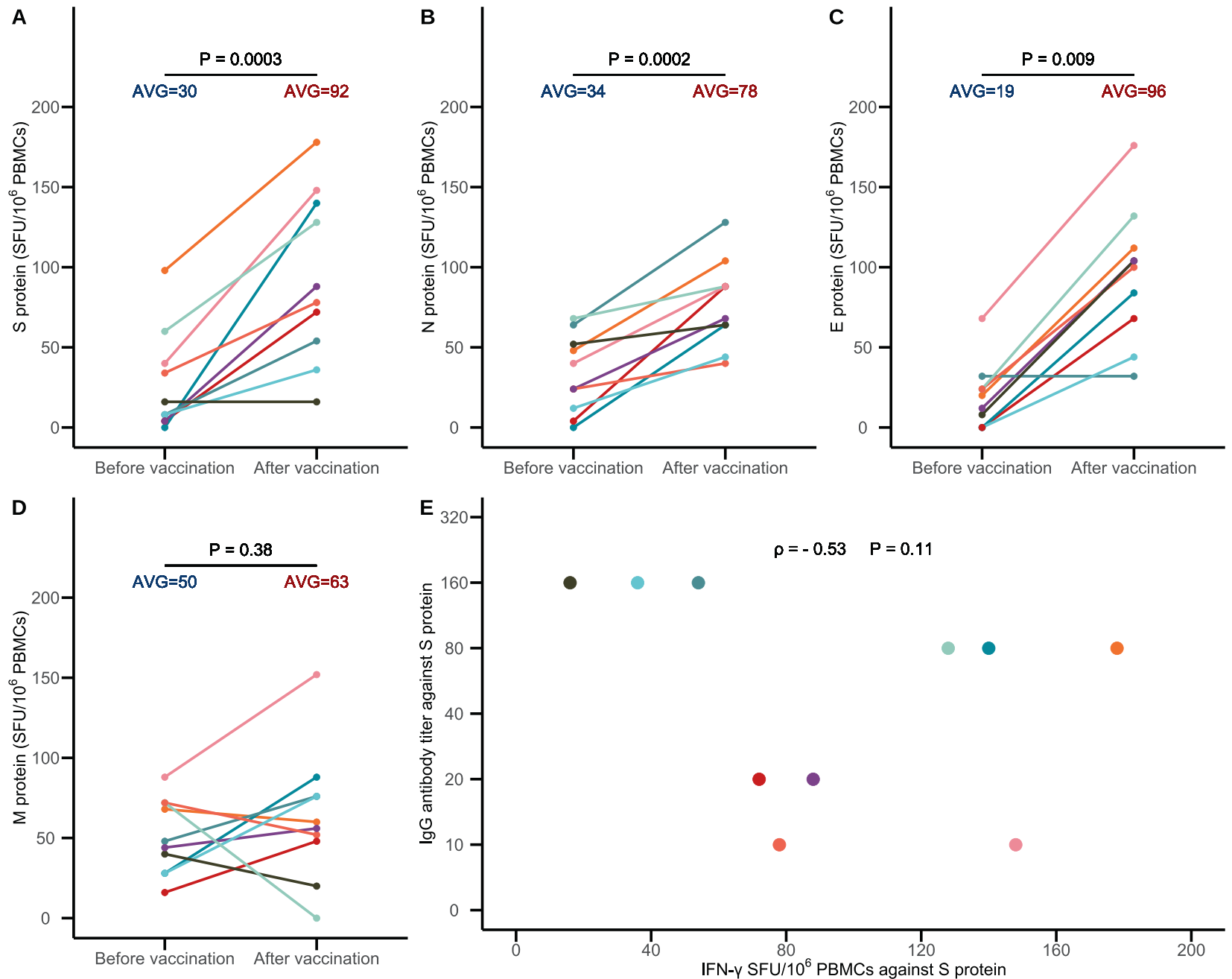

Fig. 1 T-cell responses against SARS-CoV-2 peptide pools and IgG antibody titers against the SARS-CoV-2 S protein in BBIBP-CorV recipients. A-D The T-cell responses against SARS-CoV-2 spike (S), nucleocapsid (N), envelope (E), and membrane (M) peptide pools, respectively. The Tcell response was measured as IFN- $\gamma$ spot forming units (SFU) per $10^{6}$ peripheral blood mononuclear cells (PBMCs). The mean IFN- $\gamma$ SFU per $10^{6}$ PBMCs was obtained and compared before and after vaccination. The differences were tested using paired t tests (A, B, and D) or Wilcoxon signed-rank tests (C). The $P$ value is given (horizontal line) for each comparison. Panel $E$ shows the correlation between T-cell responses and IgG antibody titers against the SARS-CoV-2 S protein in BBIBP-CorV recipients 12 weeks after the second dose. Spearman's correlation coefficient was used to determine the correlation between the IgG antibody titer and IFN- $\gamma$ SFU against the S protein. All the data in Fig. 1A-E from the 10 persons are labeled with different colors

\section{COMPETING INTERESTS}

The authors declare no competing interests.

\section{ADDITIONAL INFORMATION}

Supplementary information The online version contains supplementary material available at https://doi.org/10.1038/s41423-021-00730-8.
Correspondence and requests for materials should be addressed to W.T.

Reprints and permission information is available at http://www.nature.com/ reprints 